\title{
Small-Cage Laboratory Trials of Genetically-Engineered Anopheline Mosquitoes
}

\author{
Rebeca Carballar-Lejarazú ${ }^{1}$, Thai Binh Pham ${ }^{2}$, Vanessa Bottino-Rojas ${ }^{1}$, Adriana Adolfi ${ }^{1,3}$, Anthony A. James ${ }^{1,2}$ \\ ${ }^{1}$ Department of Microbiology \& Molecular Genetics, University of California, Irvine ${ }^{2}$ Department of Molecular Biology \& Biochemistry, University of \\ California, Irvine ${ }^{3}$ Vector Biology Department, Liverpool School of Tropical Medicine
}

\section{Corresponding Author}

\section{Anthony A. James}

aajames@uci.edu

\section{Citation}

Carballar-Lejarazú, R., Pham, T.B., Bottino-Rojas, V., Adolfi, A., James, A.A. Small-Cage Laboratory Trials of Genetically-Engineered Anopheline Mosquitoes. J. Vis. Exp. (171), e62588, doi:10.3791/62588 (2021).

\section{Date Published}

May 1, 2021

DOI

$10.3791 / 62588$

URL

jove.com/video/62588

\section{Abstract}

Control of mosquito-borne pathogens using genetically-modified vectors has been proposed as a promising tool to complement conventional control strategies. CRISPR-based homing gene drive systems have made transgenic technologies more accessible within the scientific community. Evaluation of transgenic mosquito performance and comparisons with wild-type counterparts in small laboratory cage trials provide valuable data for the design of subsequent field cage experiments and experimental assessments to refine the strategies for disease prevention. Here, we present three different protocols used in laboratory settings to evaluate transgene spread in anopheline mosquito vectors of malaria. These include inundative releases (no gene-drive system), and gene-drive overlapping and non-overlapping generation trials. The three trials vary in a number of parameters and can be adapted to desired experimental settings. Moreover, insectary studies in small cages are part of the progressive transition of engineered insects from the laboratory to open field releases. Therefore, the protocols described here represent invaluable tools to provide empirical values that will ultimately aid field implementation of new technologies for malaria elimination.

\section{Introduction}

Strategies based on genetically-engineered mosquitoes are being pursued to control transmission of vector borne pathogens such as those that cause malaria ${ }^{1}$. These include technologies 1) aimed at decreasing the numbers and densities of Anopheles mosquitoes (population suppression), or 2) aimed at impairing the ability of vectors to transmit parasites responsible for human disease (population modification, replacement, or alteration) wherein strains of vectors are engineered to express effector genes that prevent pathogen transmission. These genetic approaches have been bolstered by the advent of CRISPR/Cas9-based gene drives, with proofs-of-concept in parasite-transmitting 
mosquitoes of effective spread of payload traits as well as anti-parasitic effector molecules in caged populations.

Small laboratory cage trials represent a first step for evaluating the characteristic of transgenic strains as part of a phased approach to their further development towards field applications ${ }^{2}$. Specific outcome considerations include heritability of the introduced DNA in a competitive environment, penetrance and expressivity of the phenotype, and stability. Relevant experimental design features include the size of the cages, mosquito densities, number of replicates, overlapping or non-overlapping generations, agestructured target populations, single or multiple releases of engineered strains, male-only, female-only or mixed-sex releases, release ratios, blood meal sources (artificial or live animal), and screening procedures.

We describe here protocols used to evaluate strains of anopheline mosquitoes for inundative releases (no genedrive system) and those that carry autonomous gene-drive systems mediated by Cas9 endonucleases and guide RNAs (gRNA). Applications of these protocols appear in Pham et al. $(2019)^{2}$, Carballar-Lejarazú et al. $(2020)^{3}$, and Adolfi et al. $(2020)^{4}$.

Inundative release trials evaluate the spreading rate of a designed transgene under Mendelian inheritance following multiple releases of a large number of transgenic mosquitoes into a wild population. Without the attachment of the transgene to a drive system, data from inundative release trials provide information regarding the fitness and dynamic of the transgene of interest in a stabilized population.

When mosquito populations contain an autonomous genedrive system, small cage trials are designed to assess the dynamics of the spread of the desired transgene by determining the rate of dominant marker increase following a single introduction of transgenic males. Autonomous genedrive elements carry the genes encoding the Cas9 nuclease, gRNA and dominant marker linked in such a fashion as to be active in subsequent generations.

'Overlapping' generations refer to the simultaneous presence of multiple generations in the same cage to create an age-structured continuous population, while 'non-overlapping' refers to single discrete generations in each consecutive caged population ${ }^{2}$. Gene-drive cage experiments can be terminated once the initial dynamics of the drive (conversion) rate can be determined (8-10 generations depending on the construct), and while they provide information on the shortterm stability of the transgene within the mosquito population, they may not reveal what happens when and if the dominant marker frequencies reach or are close to full introduction (every mosquito carrying at least one copy of the gene-drive system).

\section{Protocol}

\section{Animal ethics statement}

This study was carried out in strict accordance with the recommendations in the Guide for the Care and Use of Laboratory Animals of the National Institutes of Health. Protocols were approved by the Institutional Animal Care and Use Committees of the University of California (Animal Welfare Assurance Numbers A3416.01).

\section{Inundative release trials on non-gene drive mosquitoes (Figure 1)}

\section{Cage setup and maintenance}


1. Set up three sets of triplicate $0.216 \mathrm{~m}^{3}$ cages by adding 60 second-instar wild-type (WT) larvae in each cage over three successive weeks.

NOTE: It is not possible to determine the sex of second-instar larvae by light microscopy, so the samples added to each cage will consist of both males and females.

2. At every week, provide adult females in each cage with anesthetized mice as bloodmeal source (Figure 2A) and an oviposition container 3 days after the bloodmeal.

NOTE: While an alternative artificial feeding apparatus can be used, providing live anesthetized mice for bloodmeals results in better mosquito feeding performance in these large $\left(0.216 \mathrm{~m}^{3}\right)$ cage formats. This requires an approved animal use protocol and relevant (e.g., Institutional Animal Care and Use Committee, IACUC) approval for using mice.

NOTE: Individual mice are anesthetized with a mixture of $4 \mathrm{mg} / \mathrm{mouse}$ ketamine $\mathrm{HCl}$ and $0.4 \mathrm{mg} /$ mouse xylazine. Animals are injected with between $0.1-0.5 \mathrm{~mL}$ of this mixture.

3. Hatch eggs from each cage weekly and select 60 second-instar (L2) larvae at random to be returned to their respective cages to offset mortality (weeks 4-8).

NOTE: Steps 1.1 .1 to 1.1 .3 are necessary to establish a stable and distributed age-structured population in the cages - referred to as 'Initial Phase'.

4. At week 9, assign cages assembled in step 1.1.1 randomly in triplicates for releases of the desired male release ratio.
1. Designate one set of triplicate cages as controls to assess consistency throughout the experiment.

2. Designate one set of triplicates for each desired release ratio (for example, 1:1 or 1:0.1 transgenic:WT males).

NOTE: This point on is referred to as 'Experimental Phase'.

2. Replicates and release ratios

1. Add 60 WT pupae ( 30 males and 30 females) to the control cages weekly.

2. To maintain a 1:1 ratio, add weekly 30 transgenic male pupae along with 60 (30 male and 30 female) WT pupae into each respective cage.

3. To maintain a 1:0.1 ratio, add weekly 300 transgenic male pupae along with 60 (30 male and 30 female) WT pupae into each respective cage.

NOTE: Continued addition of wild mosquitoes to the cages maintains the cage density, which is expected to diminish weekly due to age-related adult mortality.

3. Screening of phenotypes

1. Select a total of 300 larvae from each cage at random. With the use of a stereo microscope equipped with fluorescence filters, screen for the expression of the fluorescent dominant marker at the larval and pupal stages and score the sex of the resulting adults (Figure 3 ).

NOTE: The phenotypical screening will depend on the dominant marker included in the transgene construct integrated into the mosquitoes (for example, Discosoma sp. red fluorescent protein [DsRed], cyan fluorescent protein [CFP], green 
fluorescent protein [GFP]), and on the promoter driving its expression (the most used in mosquito transgenesis is the $3 \times \mathrm{P} 3$ promoter driving expression in the eyes and nerve cord).

2. Follow this protocol for as many generations as required by the outcome parameters defined in the experimental design.

NOTE: The trial is usually terminated when all mosquitoes have at least one copy of the transgene (determined by the presence of the dominant fluorescent marker) or the ratio of transgenic-to-WT mosquitoes in a cage is stabilized and does not fluctuate greatly after a few (3-5) generations.

\section{Overlapping generation trials of gene-drive mosquitoes (Figure 4)}

NOTE: Mosquitoes carrying gene-drive systems require written and reviewed protocols and should be approved by an Institutional Biosafety Committee (IBC) or equivalent, and others where required. Mosquito containment (ACL $2^{+}$level) should follow recommended procedures ${ }^{5,6,7}$. Specifically, the gene drive experiments should employ two stringent confinement strategies. The first is usually physical barriers (Barrier Strategy) between organisms and the environment. This requires having a secure insectary and standard operating procedures (including monitoring) for ensuring that mosquitoes cannot escape. The second confinement strategy can be Molecular, Ecological or Reproductive ${ }^{5}$.

\section{Cage setup}

1. Set up two sets of triplicate $0.216 \mathrm{~m}^{3}$ cages for each desired transgenic:WT male release ratio.

1. To achieve a male release ratio of $1: 1$, add 120 transgenic males, 120 WT males and 120
WT females at the pupa stage to each replicate cage.

2. To achieve a release ratio of $1: 10$, add 12 transgenic males, $120 \mathrm{WT}$ males and $120 \mathrm{WT}$ females at the pupa stage to each replicate cage.

NOTE: Different release ratios can be tested $(1: 1,1: 3,1: 10$, etc.) and the number of mosquitoes used to initiate the experiments varies accordingly. However, it is important to consider the effects of low numbers on the statistical evaluation of the data.

2. Population maintenance and screening

1. Provide 4-7 days old females in each cage with a blood meal using anesthetized mice (Figure 2A).

2. Three days after the blood meal, insert an oviposition container in each cage.

3. Hatch eggs in a larval tray, select $\sim 240$ first instar (L1) larvae at random from each cage, rear them to adulthood, and return them to their respective cages.

4. Provide additional (2-3) blood meals every 3-4 days for the newly emerged adults as described in step 2.2.1.

NOTE: No additional transgenic males are added during any of the subsequent generations.

5. Select a total of 300 larvae from each cage at random and screen them for the presence of the dominant marker phenotype at the larval and pupal stages using a fluorescence stereo microscope and score emerging adults for sex (Figure 3).

NOTE: As before, the phenotypical screening will depend on the dominant marker and promoter included in the gene-drive system and integrated 
into the transgenic mosquitoes (e.g., DsRed, CFP or GFP). If homozygous or heteroallelic disruptions of the targeted genes result in a visible phenotype (for example, genes related to eye pigmentation), screening of this trait will depend on which stage it is easiest to visualize the altered phenotype.

6. Follow this protocol for as many generations as required by the outcome parameters defined in the experimental design.

NOTE: Each generation (delimited by the blood meal) takes three weeks. The trial is usually terminated when all mosquitoes are deemed homozygous for the gene-drive construct or the populations stabilize at a maximum percentage of mosquitoes carrying at least one copy of the genedrive construct.

\section{Non-overlapping generation trials of gene-drive mosquitoes (Figure 5).}

1. Cage setup

1. Set up triplicate $0.005 \mathrm{~m}^{3}$ cage populations for each specific release ratio of transgenics to WT males to be investigated (for example, three sets of triplicate cages each set up with 1:1, 1:3, 1:10 release ratios). Set up all cages with an equal total number of males and females.

NOTE: The Supplementary File is a video demonstrating the construction of the $0.005 \mathrm{~m}^{3}$ colony cage.

1. Add 50 transgenic males, 50 WT males, and 100 WT females to each of three replicate cages to achieve a 1:1 male release ratio.
2. Add 25 transgenic males, 75 WT males, and 100 WT females to each of three replicate cages to achieve a 1:3 male release ratio.

3. Add 9 transgenic males, 90 WT males, and 100 WT females to each of three replicate cages to achieve a 1:10 male release ratio.

NOTE: Different release ratios can be tested and the number of mosquitoes used to initiate the experiments can vary accordingly. However, it is important to consider the impact of low numbers of mosquitoes on the statistical analyses. These are single releases; no additional transgenic males are added at any subsequent generation.

2. Population maintenance and screening

1. Provide the 4-7 days old females in each cage with blood meals using an artificial feeding apparatus (Figure 2B) on two consecutive days.

NOTE: Routine blood meals for females consist of a commercially available source of blood (e.g., calf's blood) provided from a feeding apparatus. Live anesthetized mice are used only to provide bloodmeals in larger $\left(0.216 \mathrm{~m}^{3}\right)$ cage formats for better feeding performance.

2. Add an oviposition container 3 days after the second bloodmeal. After three days, remove the oviposition containers.

NOTE: At this step, 5-10 females can be selected at random from each cage and placed individually in vials to assess additional fitness parameters, such as fertility and fecundity, if needed. 
3. Score by sex all adults (dead and alive) remaining in the cage and store them at $-80^{\circ} \mathrm{C}$ for molecular analysis.

4. Hatch eggs and randomly select 200 L1 larvae from the $1: 1$ and $1: 3$ ratio cages to populate new cages for the next generation.

NOTE: Due to the low frequency of starting transgenic individual in the 1:10 ratio cages, random sampling may lead to excessive loss of transgenic progeny in the next generation to carry on the population.

5. To ensure an accurate sampling for the 1:10 cages and sufficient numbers of transgenic mosquitoes, screen all larvae for the dominant marker and select 200 larvae reflecting the observed transgene frequency to populate the new cages.

NOTE: The 1:10 cages can be maintained identically to $1: 1$ and $1: 3$ cages when they reach a transgene frequency of $\geq 80 \%$.

6. Select 500 larvae from each cage at random for an in-depth analysis. Screen under a fluorescence stereo microscope for the expected marker phenotypes at the larval and pupal stages and score sex of adults (Figure 3).

NOTE: 'Exceptional' phenotypes can be selected to be further crossed and analyzed molecularly to monitor resistant allele formation.

7. This protocol can be followed for as many generations as required by the outcome parameters defined in the experimental design.

NOTE: Each generation is delimited by the bloodmeal and takes $\sim$ three weeks. The trial is usually terminated when all mosquitoes are deemed homozygous for the gene-drive construct or populations stabilize at a maximum prevalence of transgenic mosquitoes. And as before, screening for phenotypes will depend on the dominant markers and promoter integrated in the transgenic mosquitoes (for example, DsRed, CFP, GFP) or in the targeted genes if they present a visible phenotype (for example, genes related to eye pigmentation).

\section{Representative Results}

Transgenic anopheline mosquitoes generated to bear nongene drive or autonomous gene-drive modifications are set up for cage trials as described in the Protocols section. The representative results shown here depict the phenotype dynamics of the best-performing replicates of each of the cage trials experiments performed by Pham et al. $(2019)^{2}$ for Anopheles stephensi mosquitoes. The three trials (1 - 3, respectively: inundative non-gene drive, overlapping genedrive and non-overlapping gene-drive) varied in different parameters, such as the size of the cage $\left(0.216 \mathrm{~m}^{3}\right.$ vs 0.005 $\mathrm{m}^{3}$ ), whether or not the target population was age-structured, source of blood meal (mice or artificial feeder) and release ratios. As a means of representation, Figure 6 displays the observed data selected from the same release ratio (1:1) for all three protocols used, on the course of seven generations.

The 1:1 non-drive release reaches $>80 \%$ transgene introduction within 6-7 generations. For gene-drive transgenic cage trials, the $1: 1$ releases in both the non-overlapping and overlapping protocols reach this level within 3-4 generations, thus, validating the expectation that a single release of a gene drive system can be more efficient than non-drive inundative releases for transgene introduction. The faster trajectory can also be confirmed by the slope of the trendlines. Both gene- 
drive protocols, despite different set ups, present similar angles and slope trends. At the end of observation, non-drive cages achieve $\sim 80 \%$ of individuals bearing the transgene, while cages with gene-drive individuals reach complete (or near complete) introduction. Complete data and processing details on individual experiment results using the protocols described here can be found in Figures 1-3 of Pham et al. $(2019)^{2}$, Figures 2-3 of Carballar-Lejarazú et al. $(2020)^{3}$ and Figure 3 of Adolfi et al. $(2020)^{4}$.

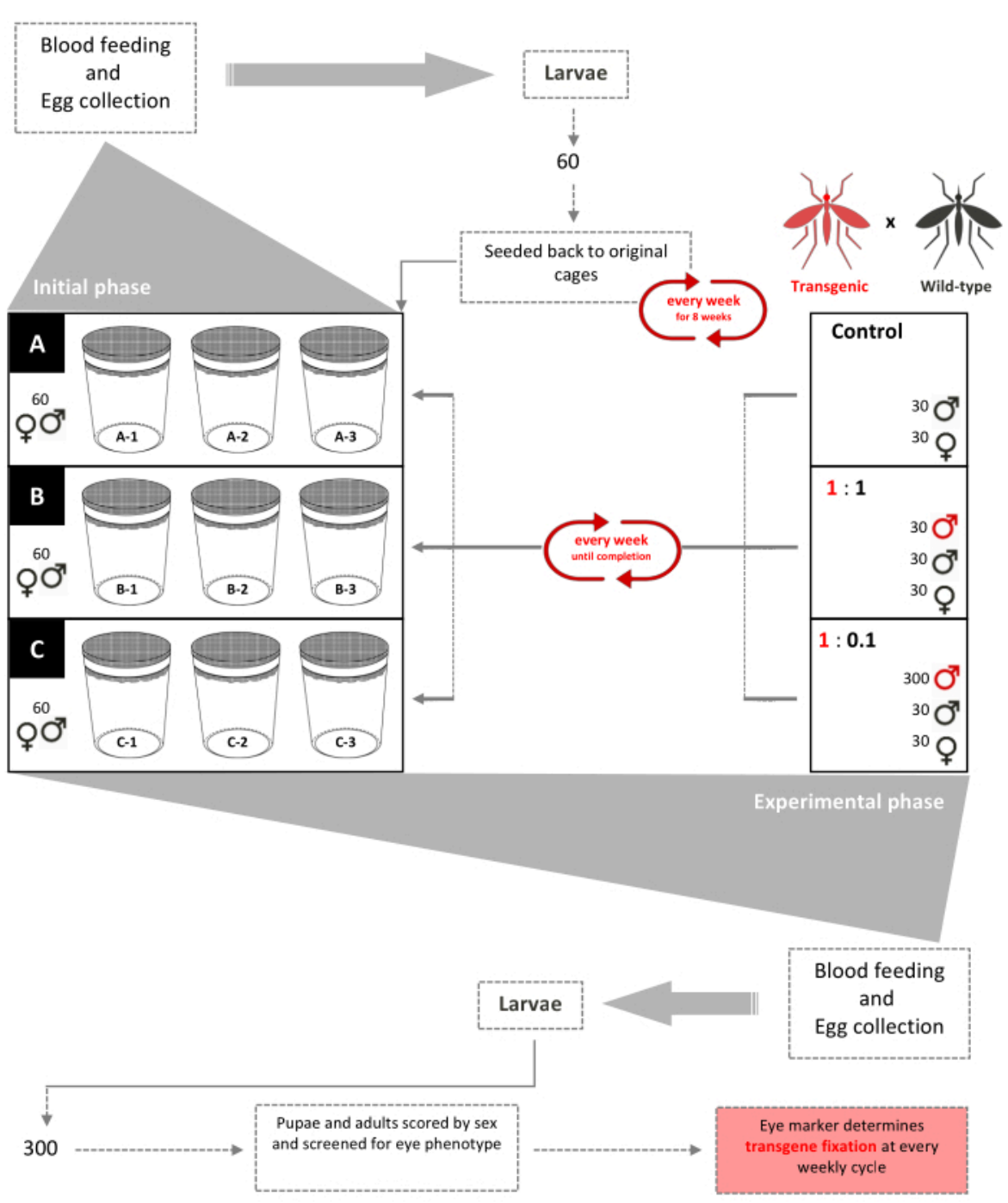

Figure 1. Non-drive inundative release trial schematic. Nine $0.216 \mathrm{~m}^{3}$ cages are set up with 60 wild-type second-instar (mixed-sexes) larvae added to each. Beginning week 3, females are provided a bloodmeal weekly and eggs are collected and hatched. Until week 8, 60 larvae are randomly selected and returned to their respective cages weekly to create an age-structured population in the cages (initial phase). Beginning week 9, the nine cages are randomly assigned in triplicate according to their transgenic:wild-type male release ratios (experimental phase). Cages $\mathrm{A}$ (Control) have no transgenic pupae added. Females are provided a bloodmeal weekly and eggs are collected, hatched, and reared to pupae. 30 male 
and 30 female wild- type pupae are added back to their cages. Cages 1:1 have an additional 30 transgenic male pupae added. Cages 1:0.1 have an additional 300 transgenic male pupae added. 300 larvae from each of the 9 cages are selected randomly and screened for the fluorescent marker. This procedure was repeated weekly until transgene fixation (stabilized ratio of transgenic-wildtype mosquitoes after a few generations). Adapted from Pham et al. (2019) ${ }^{2}$. Please click here to view a larger version of this figure.

A

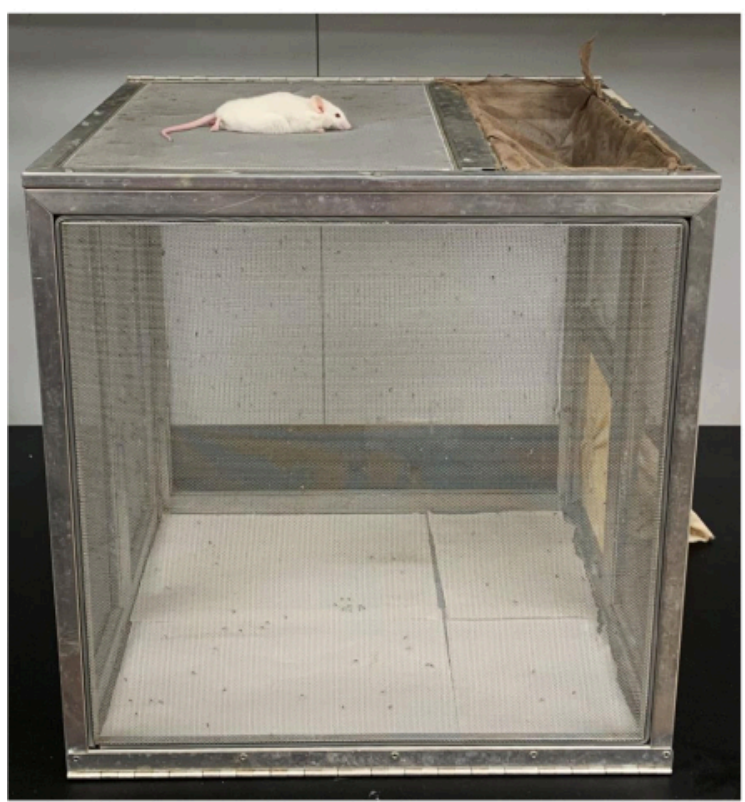

B

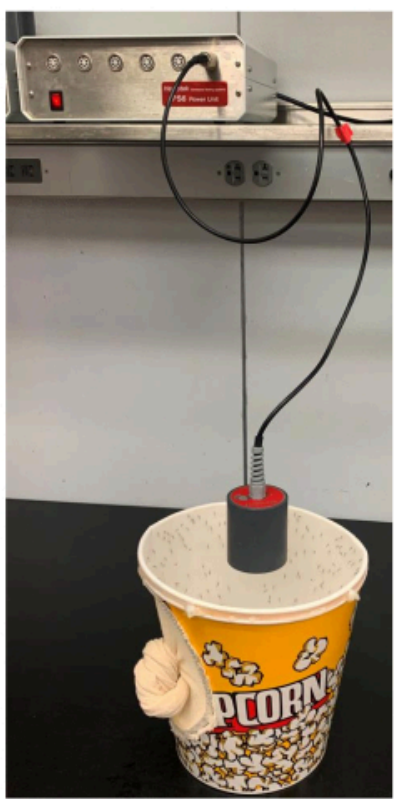

Figure 2. Blood feeding of cage populations. (A) Anesthetized mice or (B) Hemotek blood feeders are offered for blood feeding female mosquitoes on the $0.216 \mathrm{~m}^{3}$ cages or the small $0.005 \mathrm{~m}^{3}$ cages, respectively. Please click here to view a larger version of this figure. 


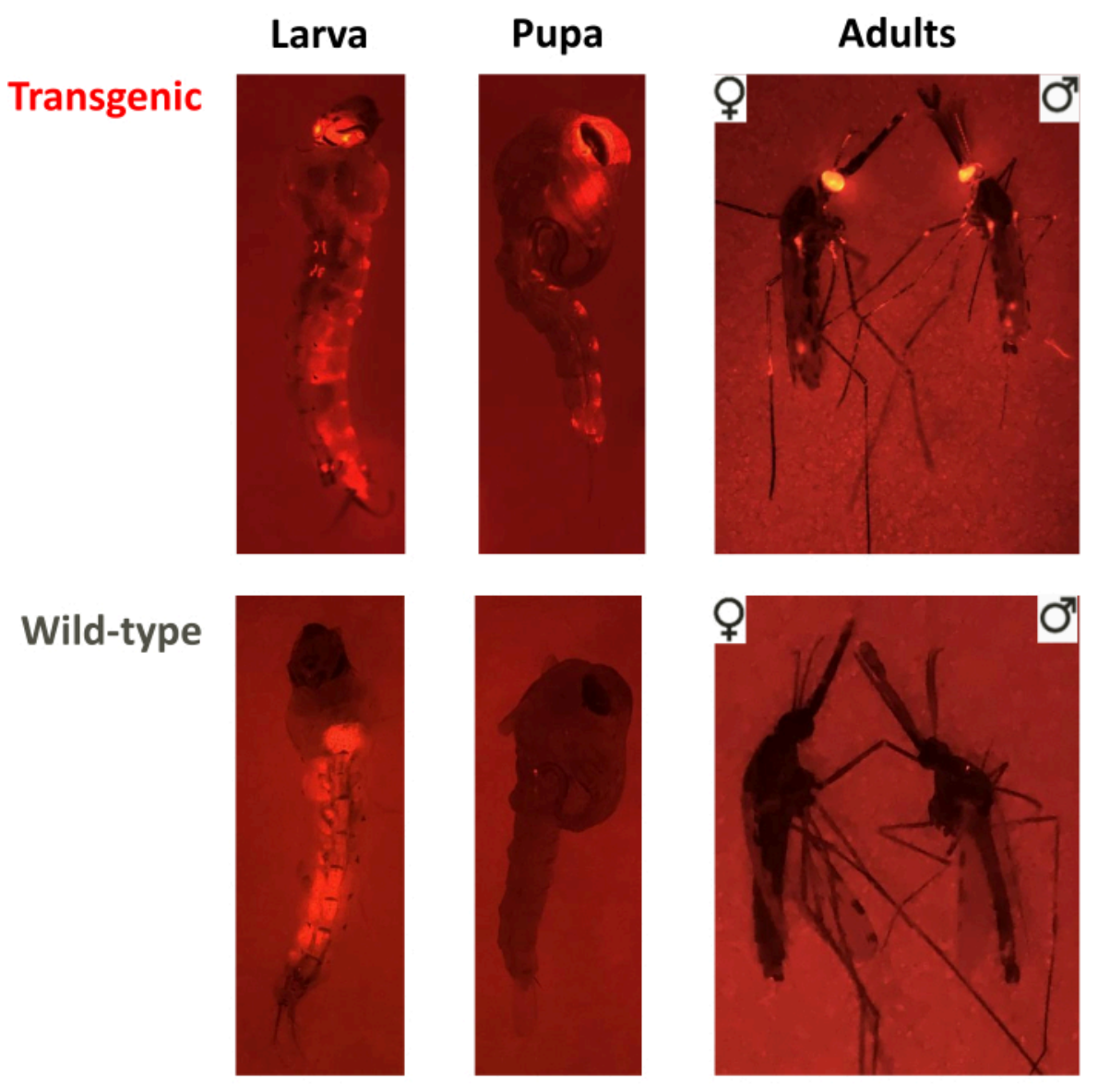

Figure 3. Screening phenotypes for non-drive, overlapping gene-drive and non-overlapping gene-drive cage trials.

Fluorescent images of a larva, pupa and adult of transgenic or wild-type phenotypes. In this example, An. stephensi individuals were screened for the DsRed marker driven by the 3xP3 promoter in the eyes (DsRed+ or DsRed-), visible in all three stages, and adults were screened for $\operatorname{sex}(\phi$ or $\widehat{\partial})$. Note the background fluorescence in wild-type larvae associated with the food bolus in the midgut. Please click here to view a larger version of this figure. 


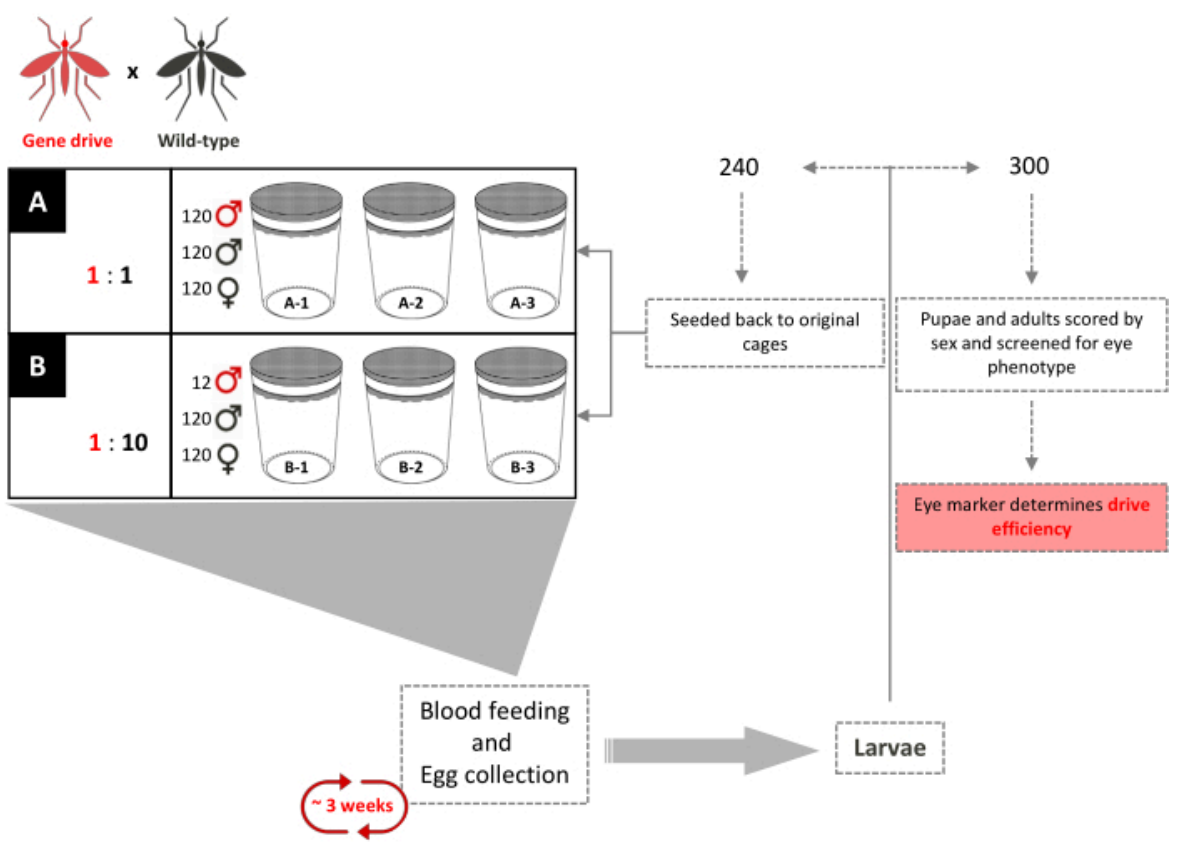

Figure 4. Overlapping gene-drive cage trial schematic. Six $0.216 \mathrm{~m}^{3}$ cages are set up in triplicate according to their gene-drive:wild-type male release ratios. 120 wild-type males and 120 wild-type females were added to each cage. Cages with a 1:1 gene-drive male release ratio had an additional 120 transgenic males added. Cages with a 1:10 male release ratio had an additional 12 transgenic males added. Until full introduction of the transgene, every 3 weeks, adult females are provided with bloodmeals and eggs are collected and hatched. A total of 240 larvae were selected randomly and returned to their respective cages. Three-hundred (300) larvae are selected randomly and screened for the dominant marker. They are later screened as pupae and adults for eye-color and sex. No additional transgenic males are added to the original cages. Adapted from Pham et al. (2019)². Please click here to view a larger version of this figure. 


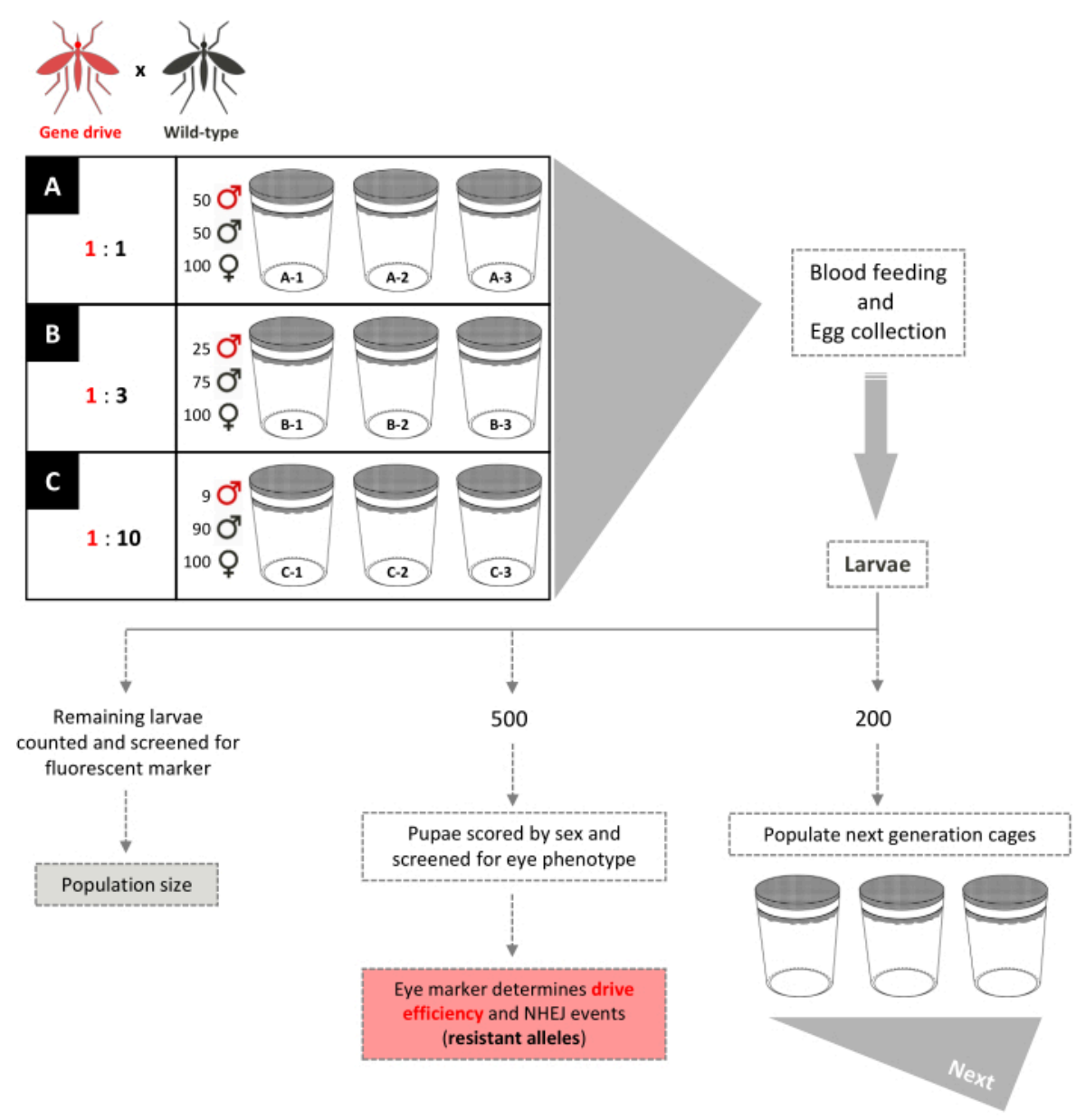

Figure 5. Non-overlapping gene-drive cage trial schematic. Nine small $0.005 \mathrm{~m}^{3}$ cages are set up in triplicate according to their gene-drive:wild-type male release ratios. Cages with a 1:1 male release ratio have 100 wild-type females, 50 wildtype males, and 50 gene-drive males added. Cages with a 1:3 male release ratio have 100 wild-type females, 75 wild-type males, and 25 gene-drive males added. Cages 1:10 male release ratio have 100 wild-type females, 90 wild-type males, and 9 gene-drive males added. Females are provided a blood meal and eggs collected and hatched. For 1:1 and 1:3 cages, 200 larvae are selected randomly and used to populate new cages, separate from that of their parents, for the next generation. An additional 500 larvae are selected randomly and reared to pupae, when they are screened for the dominant marker gene. The 500 pupae are then reared to adults and scored by sex. All remaining larvae are screened for the marker. For the 1:10 cages, all larvae are scored in generations 1-12 and 200 larvae reflecting the existing transgene frequency are used to populate new cages. Beginning at generation 13, these cages are set up identically to the 1:1 and 1:3 cages. Adapted from Pham et al. (2019) ${ }^{2}$ and Carballar-Lejarazú et al. (2020) ${ }^{3}$. Please click here to view a larger version of this figure. 


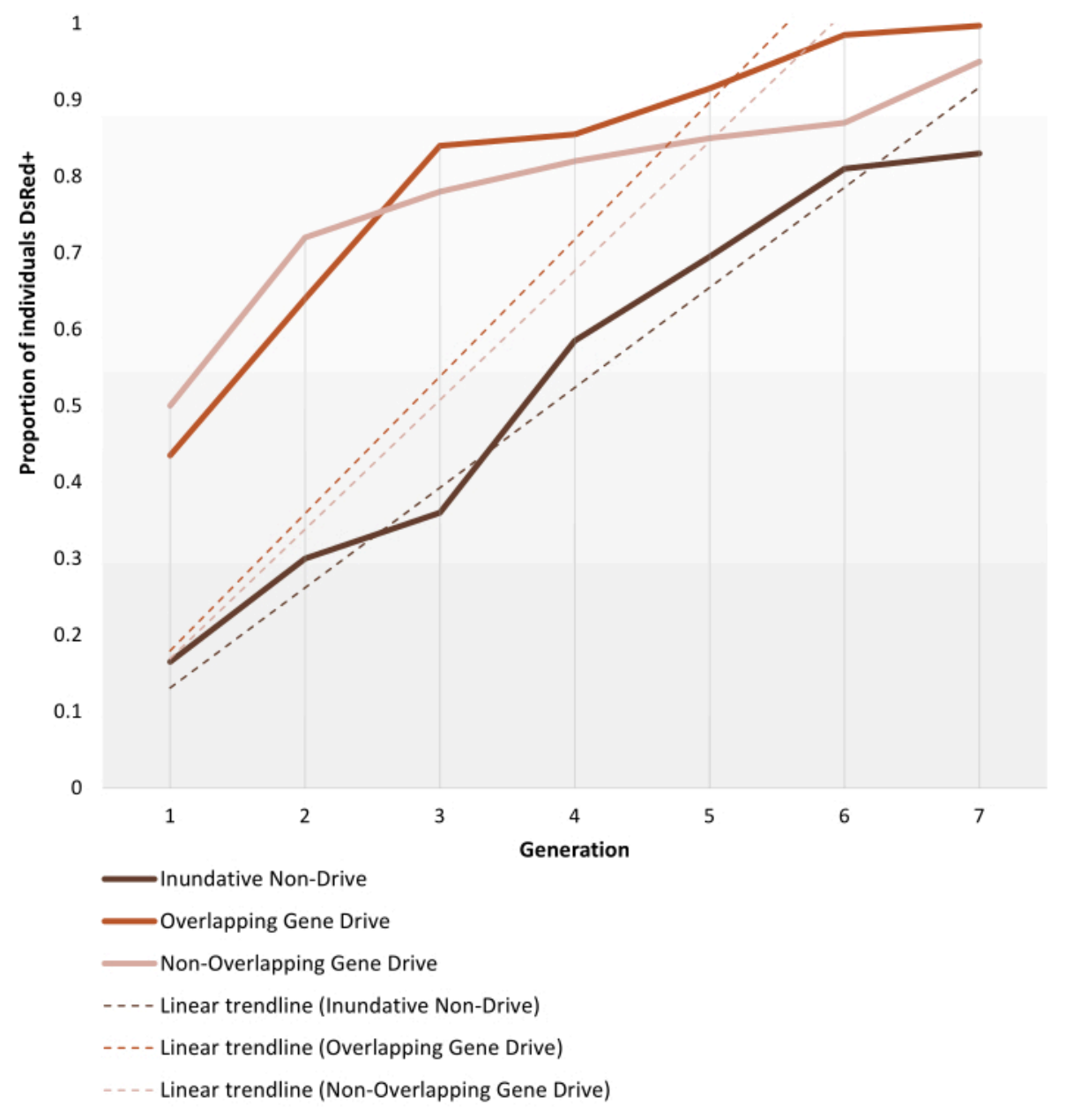

Figure 6. Predicted transgene fixation dynamics for the different population replacement cage trials. Representation of the expected phenotype dynamics of the best-performing replicates for each of the cage trials experiments performed by Pham et al. (2019) ${ }^{2}$, monitored over 7 generations. Experiments set ups are described in the Protocols. The predictions are based on data from all 9 experiments on the 1:1 release models (triplicate replicates for each of the three different cage trial protocols). The $\mathrm{X}$-axis is the generation number after initial introduction and the $\mathrm{Y}$-axis is the proportion of larvae showing the DsRed marker phenotype (DsRed+) over time. Dashed lines represent linear trendlines of the data. The DsRed+ phenotype results from having at least one copy of the modified allele. Hence the results reflect the spread of the transgene, expedited in the gene drive system, reaching (near) full introduction at the end of the observation. For the variability between replicates and full detailed data on the experiments, please refer to Pham et al. (2019) ${ }^{2}$, Carballar-Lejarazú R et al. (2020) ${ }^{3}$ and Adolfi A et al. (2020) ${ }^{4}$. Images adapted from Pham TB et al. (2019) Experimental population modification of the malaria vector mosquito, Anopheles stephensi. PLOS Genet 15(12): e1008440. doi: 10.1371/journal.pgen.1008440, Adolfi A et al. (2020) Efficient population modification gene-drive rescue system in the malaria mosquito Anopheles stephensi. Nat Commun 11(1): 5553. doi: 10.1038/s41467-020-19426-0 and Carballar-Lejarazú R et al. (2020) Next-generation gene drive for population 
modification of the malaria vector mosquito, Anopheles gambiae. Proc Natl Acad Sci USA 117(37):22805-22814. doi: 10.1073/pnas.2010214117. Please click here to view a larger version of this figure.

Supplemental File: The construction of the $0.005 \mathrm{~m}^{3}$ colony cage. Please click here to download this File.

\section{Discussion}

Genetically-engineered mosquitoes that have pathogen blocking ability or bear sterility genes constitute new tools to control vector-borne diseases. Given the multiplicity of parameters that comprise these alternative approaches, a critical step in their research consists of laboratory-confined experimental evaluations that allow a fast and safe prediction of the potential outcomes of a synthetic transgene release for control purposes ${ }^{1}$.

Because the monitoring of the transgene dynamics in caged populations can extend for several months, one of the central aspects of the protocols is the consistency in experimental design between replicates (including mosquito rearing, cage size, age-structured populations, fixed release ratios, stable blood meal sources and minimally invasive screening procedures).

Male-only releases are considered ideal because male mosquitoes neither transmit pathogens nor feed on humans, therefore they can safely introduce heritable characteristics into wild populations. In laboratory cage experiments, it is possible to detect transgenic strains with reduced male mating competitiveness and other fitness loads associated with transgene integration. However, direct and specific experiments, such as those conducted in large cages ${ }^{10}$, can be conducted to properly analyze male competitiveness, as well as female fecundity in more natural mosquito densities $^{2}$. Furthermore, empirical data from the cage trials can be used to parameterize models of cage population dynamics, including resistant allele formation, and provide useful information on effectiveness and possible adjustments in the proposed technology.

The protocols described here can be easily adapted to other experimental designs as required, with minimal requirements regarding regular insectary infrastructure and conditions. In addition, except for the commercial cages and microscopes, most of the materials are inexpensive and allow low-cost multiple replicates and iterations of the trials. Notably, this also allows multiple transgenic strains to be pre-screened in small cage trials in order to prioritize best-performing candidates to be moved forward in the phased testing pathway and to suspend testing on those showing suboptimal performances.

Finally, concern regarding the use of genetically modified organisms motivates the elaboration of frameworks for the development, evaluation, and application of genetic strategies for prevention of mosquito-borne diseases ${ }^{5,8,9}$. The relevance and execution of the protocols defined here are consistent with these guidelines.

\section{Disclosures}

The authors have no disclosures.

\section{Acknowledgments}

We are grateful to Drusilla Stillinger, Kiona Parker, Parrish Powell and Madeline Nottoli for mosquito husbandry. Funding was provided by the University of California Irvine Malaria Initiative. AAJ is a Donald Bren Professor at the University of California, Irvine. 


\section{References}

1. Carballar-Lejarazú, R., James, A.A. Population modification of Anopheline species to control malaria transmission. Pathogens and Global Health. 111 (8), 424-435 (2017).

2. Pham, T.B. et al. Experimental population modification of the malaria vector mosquito, Anopheles stephensi. PLOS Genetics. 15 (12), e1008440 (2019).

3. Carballar-Lejarazú, R. et al. Next-generation gene drive for population modification of the malaria vector mosquito, Anopheles gambiae. Proceedings of the National Academy of Sciences. 117 (37), 22805-22814 (2020).

4. Adolfi, A. et al. Efficient population modification genedrive rescue system in the malaria mosquito Anopheles stephensi. Nature Communications. 11 (1), 5553 (2020).

5. Akbari, O.S. et al. Safeguarding gene drive experiments in the laboratory. Science. 349 (6251), 927-929 (2015).

6. Benedict, M.Q. et al. Recommendations for Laboratory Containment and Management of Gene Drive Systems in Arthropods. Vector-Borne and Zoonotic Diseases. 18 (1), 2-13 (2018).

7. $C D C / N I H$ Biosafety in Microbiological and Biomedical Laboratories. (6th Edition), 1-574, at <https://www.cdc.gov/labs/pdf/SF_19_308133A_BMBL6_00-BOOK-WEB-final-3.pdf> (2020).

8. Adelman, Z. et al. Rules of the road for insect gene drive research and testing. Nature Biotechnology. 35 (8), 716-718 (2017).
9. Long, K.C. et al. Core commitments for field trials of gene drive organisms. Science. 370 (6523), 1417-1419 (2021).

10. Facchinelli, L., North, A.R., Collins, C.M. et al. Largecage assessment of a transgenic sex-ratio distortion strain on populations of an African malaria vector. Parasites Vectors. 12, 70 (2019). 